\title{
ASSESSMENT OF THE TOPICAL AND SYSTEMIC EFFECTS OF OMEGA-3 ON ORAL MUCOSAL WOUND HEALING IN ALBINO RATS: A HISTOLOGICAL AND BIOCHEMICAL STUDY
}

\author{
Soha Basha* and Sahar Mahmoud ElRefai***
}

\begin{abstract}
Omega-3 was recently introduced as an effective therapeutic agent and a powerful antiinflammatory agent, which promotes whole-body wound healing including ulcers as it has an important role in modulation of proinflammatory cytokine production.
\end{abstract}

Aim of study: This study was conducted to evaluate and compare the effect of topical application and systemic administration of omega-3 on the healing of oral mucosal wounds which were experimentally induced in albino rats.

Materials \& Methods: The experiment comprised 45 albino rats which were divided into 3 groups ( $\mathrm{n}=15$ per group); control group (C) not receiving any treatment, group (L) receiving topical application of omega-3, and group (S) receiving systemic omega-3, 5 rats from each group were sacrificed at day 2, 4 and 8 respectively. Tissue samples were obtained and prepared for histological and biochemical evaluations. The data obtained were statistical analyzed and compared.

Results: Biochemical results showed significant improvement regarding TNF- $\alpha$ and IL- 8 level after 4 and 8 days in local and systemic groups compared to the control. The topical omega- 3 group showed better results than the systemic with significant differences in TNF- $\alpha$ after 2 and 4 days, while for IL- 8 the difference was only significant after 8 days. The histological results in both local and systemic treated groups after 2 days revealed inflammation and epithelial degeneration which gradually reepithelized by 4 to 8 days.

Conclusion: Omega- 3 greatly enhanced oral ulcer healing and showed superior effects using topical application.

KEY WORDS: Interlukin-8, Oral ulcers,omega-3, TNF

* Lecturer Oral Medicine and Periodontology Department, Faculty of Dentistry, Pharos University in Alexandria, Egypt.

** Lecturer of Oral Pathology, Faculty of Dentistry, Fayoum University, Cairo, Egypt. 


\section{INTRODUCTION}

Ulcers are among the most frequent lesions encountered in the oral cavity, they may be manifested as either solitary or multiple lesions affecting skin and/or oral mucous membrane ${ }^{(1)}$.

An ulcer is generally defined as a break in the skin with loss or discontinuity of surface epithelium and can also be defined as a local defect due to disruption in mucosal integrity. The exact cause of ulcers remains unclear, nevertheless many factors have always been blamed including primarily trauma, moreover chemicals, infections, cancers, radiation and certain therapeutic drugs ${ }^{(2)}$.

Ulcers can be classified into either primary lesions, with early manifestation such as erosion, or secondary if they result from subsequent rupture of vesicles and bullae.The exact pathogenesis of ulcers differs vastly according to their etiology ${ }^{(3,4)}$.

Ulcers are painful sores that cause discomfort,the goal of its treatment is to relieve symptoms; therefore, finding suitable drugs with fewer side effects is the concern of many researchers. Steroids (topical and oral) and mouthwashes are usual treatments for oral ulcers; however, systemic absorption of local steroids can have undesirable influences on the immune system and may lead to secondary infections. More complex processes for such lesions are seen in some autoimmune diseases, where a number of different genetic mutations take place. ${ }^{(5)}$

In recent years, Omega- 3 has received attention being rediscovered as an effective therapeutic agent and a powerful anti-inflammatory, which promotes whole-body wound healing including ulcers ${ }^{(6,7)}$.

It is naturally found in common diet as in cod liver oil, fish oil, marine animals with a high amount of fat, such as tuna and plant oils from walnuts. Omega 3 fatty acids are bioactive lipids making an important part of cell membranes, thus they modulate membrane protein function, cellular signaling and gene expression. Considerable interest has been focused on the potential health benefits of omega-3 fatty acids on many diseases. ${ }^{(\mathbf{8 , 9})}$

It was also recently discovered that (omega-3 fatty acids) helped defend against ulcers on multiple fronts by augmenting both defensive and offensive factors thus enhancing clearance of inflammation within the lesion promoting tissue regeneration ${ }^{(\mathbf{9 , 1 0})}$.

Tumor necrosis factor alpha (TNF- $\alpha$ ) is one of a group of proinflammatory cytokines that appears rapidly after infection and injury. It has a pivotal role in withstanding pathogenic invasion ${ }^{(\mathbf{1 1}, \mathbf{1 2})}$. Similarly IL-8 referred to as neutrophil chemotactic factor (NCF) and neutrophil activating factor (NAF) is an important mediator of host response to injury and inflammation. It acts as chemo-attractant for $\mathrm{T}$ cells and is significantly increased in patients with oral ulcers $^{(13)}$.

\section{MATERIAL AND METHODS}

Animals: 45 adult male Albino rats weighting (200-250g) obtained from the animal house of Medical Research Institute, Alexandria University were used in the study. Throughout the study the animals were kept at the animal house of Faculty of Dentistry, Pharos University in polypropylene cages, 10 rats each fed normal diet, and water add libitum. The room temperature was about $22-24^{\circ} \mathrm{C}$ and the animals were exposed to 12:12 hours light dark cycles. The experimental research protocol was approved by the Ethics Review Board of Faculty of Dentistry, Pharos University.

Ulcer induction: All experimental rats were subjected to an induced ulcer on their palatal oral mucosae as follows:

The rats were fixed on their backs and anaesthetized with intravenous pentobarbital injection $(50 \mathrm{mg} / \mathrm{kg})$. In order to create an ulcer, a round $5.5 \mathrm{~mm}$ in diameter filter paper was soaked in $15 \mathrm{ml}$ 
of $50 \%$ acetic acid and pressed onto the palatal mucosa for 60 seconds $^{(7)}$.

Animals were then divided into 3 groups (15 rats in each group).

Control group $(\mathrm{C})$ : rats with induced ulcers not receiving treatment.

Local application omega-3 group (L): a swab was soaked in omega-3 soft gelatin capsules 1000mg (SEDICO-EGYPT) was packed on the ulcer throughout the study period ${ }^{(8)}$.

Systemically administered omega-3 group(S): omega-3 with a concentration of $600 \mathrm{mg} / \mathrm{kg}$ body weight was guided into the stomach by gastric gavage twice daily throughout the study period ${ }^{(7)}$.

\section{Biochemical Evaluation}

Excised palatal mucosal tissues obtained from the three experimental groups of rats were directly dissected and homogenized in appropriate buffer and then centrifuged, according to the instructions of the biochemical assay. The levels of TNF- $\alpha$ and IL- 8 were measured using an enzyme-linked immunosorbent assay ELISA commercial kit (R\&D Systems, EUA).

Data obtained were fed to the computer and analyzed using IBM SPSS software package version 20.0. The Kolmogorov- Smirnov, Shapiro and Dagstino tests were used to verify the normality of distribution of variables, ANOVA was used for comparing the three studied groups and followed by Post Hoc test (LSD) for pairwise comparison. Significance of the obtained results was judged at $\mathrm{P}<0.05$ was accepted as significant.

\section{Histological evaluation}

Histological samples were collected on post-injury days $2,4,8$ and each wound was excised maintaining approximately $3 \mathrm{~mm}$ of normal palatal mucosa around the incision, specimens were then processed for paraffin embedding and longitudinal serial sections were cut to a thickness of $6 \mathrm{um}$. The sections were mounted on glass slides then stained with hematoxylin and eosin. Two independent examiners evaluated the specimens in a blind fashion in order to estimate the tissue response in the areas adjacent to the created ulcer and compared with the normal tissues.

\section{Statistical results}

On the basis of ANOVA one way analysis test the level of TNF- $\alpha$, after 2 days showed no significant difference between the three studied groups. While, after 4 and 8 days significant difference existed between the three studied period. While after 4 and 8 days significant difference existed comparing between the three studied periods. (Table 1)

Concerning IL-8, significant difference existed between control and systemic groups and between control and topical groups after 2 and 4 days, but no significant difference existed between systemic group and topical group. However after 8 days significant difference existed between the three studied groups.

Regarding the percentage of change of TNF- $\alpha$ level within each group, significant decrease occurred from 2 to 8 days, which amounted to as high as $70 \%$ in the topical group (L) while in the systemic group (S) it decreased only by $58 \%$ and the least amount of decrease was seen in the control group (C) and was equal to $38 \%$.

Concerning the percentage of changes of IL-8 level within each group, significant decrease occurred from 2 to 8 days with the greatest decrease in the topical group (L) by $60 \%$ while in the systemic group (S) it decreased by $50 \%$ and the least amount of decrease was seen in the control group (C) and was equal to $28 \%$. 
TABLE (1): Comparison between the three studied groups according to TNF alpha and IL-8

\begin{tabular}{lccccccc}
\hline & Control & Systemic & Topical & $\mathbf{p}_{1}$ & $\mathbf{p}_{2}$ & $\mathbf{p}_{3}$ & $\mathbf{p}_{4}$ \\
\hline TNF alpha & & & & & & & \\
After 2 days & $35.7 \pm 1.4$ & $34.0 \pm 3.3$ & $35.0 \pm 2.6$ & 0.332 & 0.143 & 0.529 & 0.391 \\
After 4 days & $28.4 \pm 4.3$ & $21.0 \pm 2.6$ & $17.0 \pm 2.1$ & $<0.001^{*}$ & $<0.001^{*}$ & $<0.001^{*}$ & $<0.001^{*}$ \\
After 8 days & $22.0 \pm 3.3$ & $14.0 \pm 2.2$ & $10.4 \pm 1.5$ & $<0.001^{*}$ & $<0.001^{*}$ & $<0.001^{*}$ & $0.003^{*}$ \\
\% of change & $\downarrow 38.4$ & $\downarrow 58.8$ & $\downarrow 70.3$ & & & & \\
IL-8 & & & & & & & \\
After 2 days & $42.0 \pm 2.7$ & $38.0 \pm 2.6$ & $36.5 \pm 2.9$ & $<0.001^{*}$ & $0.003^{*}$ & $<0.001^{*}$ & 0.233 \\
After 4 days & $38.0 \pm 2.9$ & $26.5 \pm 1.7$ & $24.5 \pm 2.8$ & $<0.001^{*}$ & $<0.001^{*}$ & $<0.001^{*}$ & 0.093 \\
After 8 days & $30.0 \pm 2.9$ & $19.0 \pm 2.6$ & $14.6 \pm 2.7$ & $<0.001^{*}$ & $<0.001^{*}$ & $<0.001^{*}$ & $0.001^{*}$ \\
\% of change & $\downarrow 28.6$ & $\downarrow 50.0$ & $\downarrow 60.0$ & & & & \\
\hline
\end{tabular}

$p_{1}$ : p value for ANOVA test for comparing between the three studied groups

$p_{2}$ : p value for Post Hoc Test (LSD) for ANOVA with repeated measures for comparing between Control and Systemic $p_{3}: p$ value for Post Hoc Test (LSD) for ANOVA with repeated measures for comparing between Control and Topical $p_{4}:$ p value for Post Hoc Test (LSD) for ANOVA with repeated measures for comparing between Systemic and Topical *: Statistically significant at $p \leq 0.05$

\section{Histological results}

The experimentally produced ulcer of the control group after 2 days showed a wound of considerable size with degenerated epithelial and keratin layer. The lamina propria and submucous layers were heavily infiltrated with inflammatory cells (Fig1a). The omega-3 treated groups both local (L) and systemic (S) showed epithelial degeneration with a deep ulcer formation and complete absence of the covering keratin layer (Figs 2a \& 3a). After 4 days, the control group showed deep ulcer region with severe degenerated epithelial and subepithelial layers (Fig1b), while the locally treated group (L) showed a remarkable reduction in the wound size with an intact epithelial layer lining most of its depth while the systemic treated group (S) revealed only slight degree of regeneration of the keratinized stratified squamous epithelium of the hard palate (Figs 2b \& 3b). In both groups, moderate numbers of inflammatory cells were also noted. After 8 days the control group showed regenerated thin epithelium lining the ulcer region and remnants of granulation tissue are found. Connective tissue is infiltrated with inflammatory cells (Fig1c). Both groups (L and S) showed an even thickness of the ulcer region and complete regeneration however the locally treated $(\mathrm{L})$ groups presented full reepithelialization of epithelial ridges covered by a well keratinized surface and connective tissue papillae of normal thickness (Figs 2c \& 3c) inflammatory cells were also noted however, minimally infiltrated the subepithelial layers of the (L) group compared to those of the (S) group. 


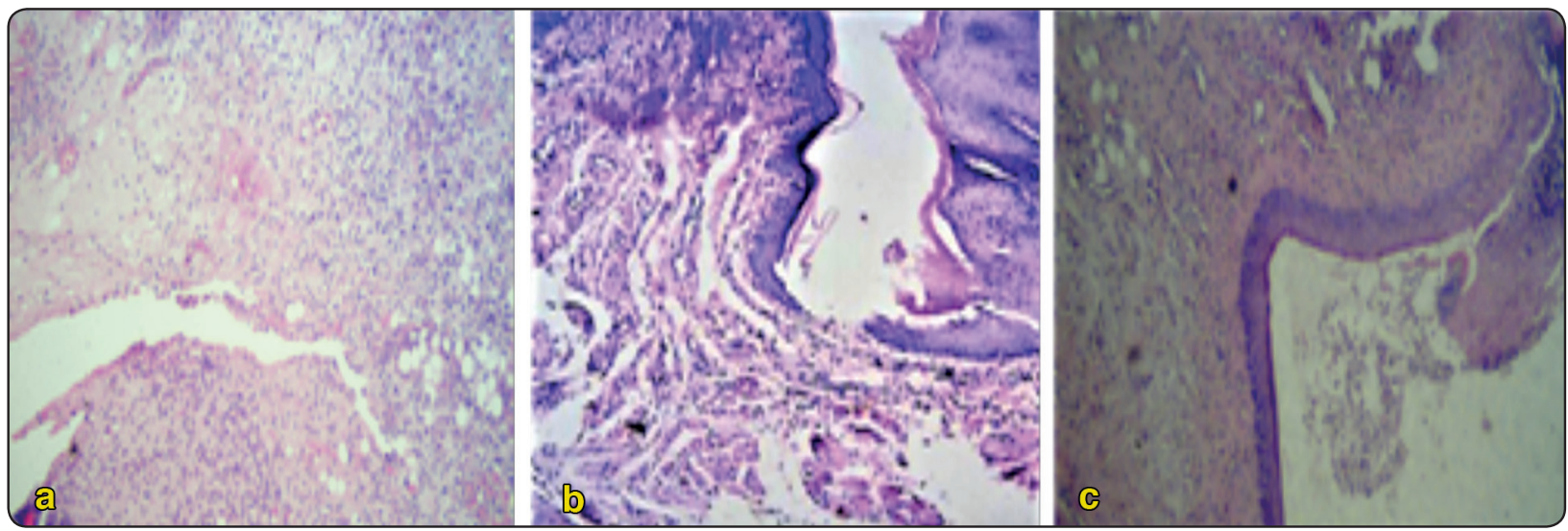

Fig. (1) Photomicrographs of the rat palate showing gradual healing of the induced wounds in the control group at days 2(1a), day $4(1 b)$, and 8 day (1c) (H\&Ex100)

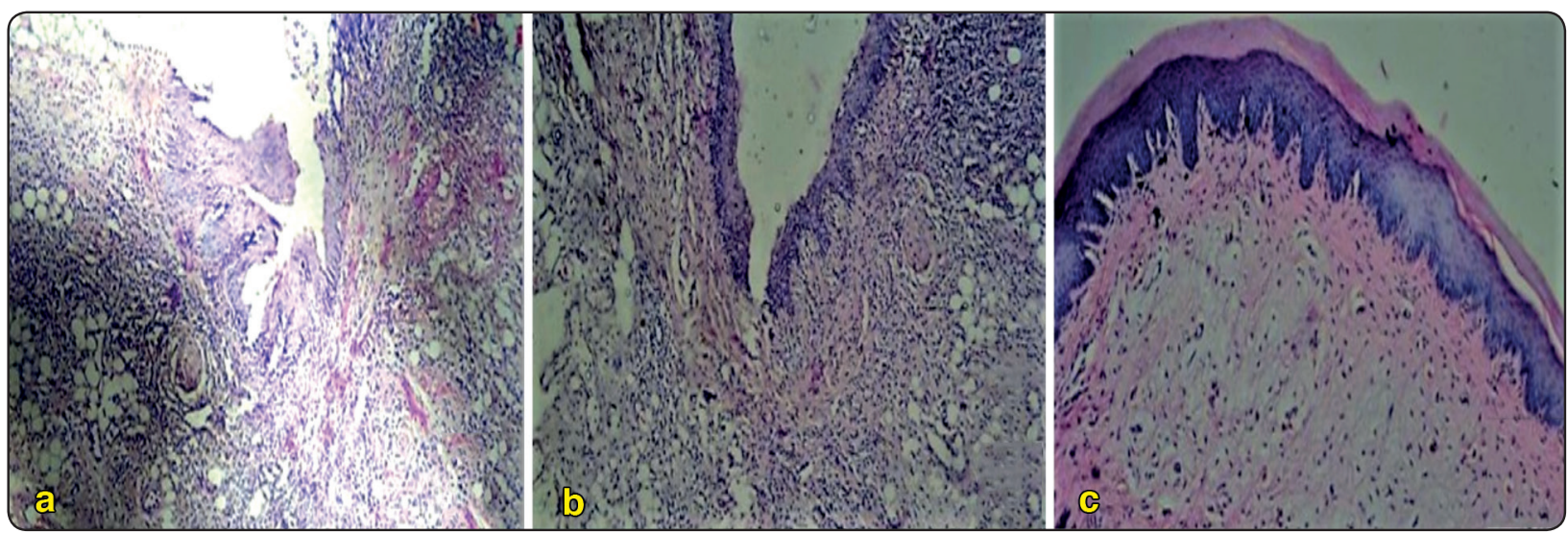

Fig. (2) Photomicrographs of the rat palate showing gradual healing of the induced wounds in the local omega-3 group at days 2(2a), day $4(2 b)$, and day $8(2 c)(H \& E x 100)$

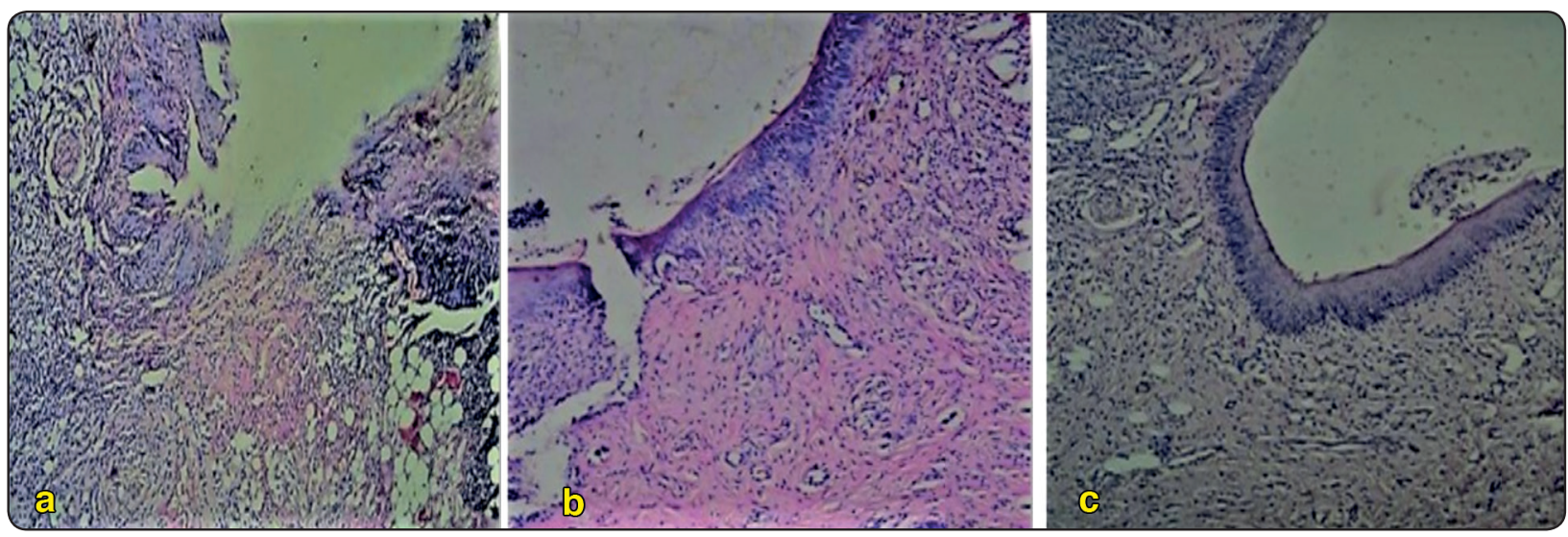

Fig. (3) Photomicrographs of the rat palate showing gradual healing of the induced wounds in the systemic omega-3 group at days 2 (3a), day 4 (3b), and day 8 (3c) (H\&Ex100) 


\section{DISCUSSION:}

Oral ulcers are among the most common manifestations of oral diseases. Being painful and annoying, treatment and management of oral ulcers is of utmost importance. Application of new treatments that are more patient compliant, less expensive, easy to use, readily available and with minimal side effects would be helpful ${ }^{(\mathbf{8})}$.

Omega-3 polyunsaturated fatty acids are one of the best prescribed therapies for such conditions, owing to their clear role in early wound epithelialization, and inhibition of many inflammatory responses reported by various studies ${ }^{(\mathbf{1 4})}$.

Experimental studies have found gastrointestinal benefits of omega-3 and has been shown to decrease the acid-secretion, significantly increasing the activity of anti-oxidant enzymes and lowering lipid peroxidation in the rat gastric mucosa ${ }^{(\mathbf{1 5 )}}$.

Its anti-inflammatory properties arise from processes such as the partial replacement of arachidonic acid in cell membranes. There is also a reduction in the chemotaxis of monocytes and neutrophils, modulating the inflammatory response in its earliest stages ${ }^{(\mathbf{1 6})}$.

TNF- $\alpha$ also known as cachectin is an inflammatory cytokine and apoptotic molecule that is recognized as a crucial contributing factorin inflammation and fibrosis. While beneficial in host defense to pathogens, in excess, TNF- $\alpha$ is associated with host damaging effects. Impaired healing in animal models and age-related delayed healing of acute human wounds exhibit raised local and systemic levels of TNF- $\alpha .^{(17,18,19)}$

Omega-3 has a protective action against the damaging effects of TNF- $\alpha$, Khosroshahi et al (20) reported in their study on hemodialysis patients a significant reduction of TNF- $\alpha$ level after two months of omega -3 treatment, they concluded that omega-3 fatty acids decrease the production of classic inflammatory cytokines as well as the expression of adhesion molecules involved in inflammatory interactions between leukocytes and endothelial cells ${ }^{(\mathbf{1 9})}$.

IL-8 is a cytokine that plays an important role in inflammation and wound healing. Qazi et $\mathbf{a l}^{(\mathbf{2 1 )}}$ reported that in many cell types, IL-8 has a capacity to recruit $T$ cells as well as nonspecific inflammatory cells into sites of inflammation by activating neutrophils. Furthermore, IL-8 stimulates deposition of collagen I and other proteins during wound healing in vivo as confirmed by Matsuyama Wet al ${ }^{(22)}$ who reported in their study on fiftychronic obstructive pulmonary disease (COPD) patients that omega-3 had a significant anti-inflammatory role in decreasing IL-8 and TNF- $\alpha$ levels ${ }^{(21,22,23)}$.

Despite this weight of evidence and the considerable current use, there is still a need for further researches, therefore this study was conducted to assess the topical and systemic effects of omega-3 on mucosal wound healing regarding oral ulcers.

The results of the present study regarding the biochemical analysis showed that there was a significant decrease in inflammation represented by the levels of IL- 8 and TNF- $\alpha$ either with the local or systemic omega 3 supplementation after days 2, 4 , and 8 , with a gradual decrease from one period to the other, and this was in agreement with Hashemipour et al ${ }^{(8)}$ who reported in their study on rats (palatal ulcers), high scores of inflammation on day 2 and 4 in control group in comparison with the consecutive days while lowest inflammatory scores on days 6 and 8. These findings were also confirmed by Thieset $\mathbf{a l}^{(24)}$ and Kang et $\mathbf{a l}^{(25)}$ who showed decrease in TNF- $\alpha$ in their reports.

The fact that omega-3 fatty acids play an important role in the regulation of inflammation can be explained through multiple pathways including, induction of several potent anti-inflammatory lipid mediators, suppression of nuclear transcription factors activity, and reduction of the pro-inflammatory enzymes and cytokines ${ }^{(\mathbf{8 , 2 4 , 2 5})}$. 
The histological results of the present study, showed severe signs of epithelial atrophy with absence of keratin layer and inflamed underlying connective tissue. With local and systemic omega -3 application, there was a gradual reduction in inflammation, increase in epithelial and connective tissue regeneration, appearance of keratin layer, and finally high vasculature, all occurring gradually throughout the period of 2,4, and 8 days, giving the best repair effect at the end of the treatment period.

These results were in accordance with Hashemipour et al ${ }^{(8)}$, where they showed that by using omega-3, the greatest reepithelialization being observed on days 6 and 8 of their study, and that the fibroblasts significantly increased throughout the whole period and promoted tissue regeneration, advocating that these fibroblasts produce PGE2, IL-6, and TNF- $\alpha$, which have a role in collagen synthesis and in turn wound closure. Similar results were reported by Hankenson et $\mathbf{a l}^{(26)}$.

On the other hand Arzu Gercek et al (27), explained the reepithelialization through monitoring TGF- $\beta$ levels, this growth factor inhibitor, which plays an important role in the healing process together with platelet derived growth factor (PDGF), they stimulate fibroblast chemotaxis, collagen production, and inhibit collagen degradation, thus, promoting fibrogenesis. This was confirmed by other studies that showed that the level of TGF increases gradually, being highest on day 6 of their study period, making tissue regeneration and healing more pronounced as time passes ${ }^{(\mathbf{8 , 2 7})}$.

Conflicting to these results, Cardoso et $\mathbf{a l}^{(28)}$, reported in their research using omega- 3, 6, and 9 that omega-3 treated group showed tendency to a delay in wound closure in the first 10 days, which disagrees not only with our results but alsowith other authors who observed faster wound repair after administration of codfish oil. This difference could be explained by the fact that codfish oil has several components or concentrations that may influence healing, while we used purified preparations ${ }^{(27)}$.

In consistence with Hashemipour et al (8), the results of the present study showed that there was a significant difference between the local and systemic supplements which was in favor of the local form regarding the decrease in inflammation and the better and faster wound healing.

These results were also supported by Terkelesn et $\mathbf{a l}^{(29)}$ who reported that omega-3 topical application significantly accelerated both the epithelial and vascular component of healing compared to the systemic supplement being clarified throughthe fact that systemic supplements need more time to heal the wound since they reach through blood unlike the direct effect that the local forms offer ${ }^{(\mathbf{8 , 2 9})}$.

\section{CONCLUSION}

The findings of this study indicate that omega-3 fatty acids ( $L$ and $S$ ) can promote healing of oral ulcers in animal models where the topical application being more effective than systemic administration. The results showed wound size reduction, increase in fibroblastic activity, re-epithelialization and granulation tissue formation. To sum up, the use of omega-3 fatty acids is safe, practical, and could be an alternative treatment for oral ulcers.

\section{ACKNOWLEDGMENT}

The authors would like to thank Dr. Nevien Mahmoud Lecturer of Biochemistry,Faculty of Dentistry, Pharos University for her valuable help in the biochemical analysis throughout this study.

\section{REFERENCES}

1. Scully and Crispian: Soreness and ulcers". Oral and maxillofacial medicine: the basis of diagnosis and treatment 2013 (3erd ed.), chapter 14. pp. 131-139.

2. Generoso $\mathrm{S}$ de $\mathrm{V}$, Rodrigues NM, Trindade LM, et al. Dietary supplementation with omega-3 fatty acid attenuates 5-fluorouracil induced mucositis in mice. Lipids in Health and Disease. 2015;14:54. 
3. Tahura Khwaja, Amsavardani Tayaar S. Review of oral ulcers: A diagnostic dilemma. Journal of Medicine, Radiology, Pathology \& Surgery. 2016; 3: 20-24.

4. Tyldesley, Anne Field, Lesley Longman and William R. Tyldesley's Oral medicine (5th ed.). Oxford: Oxford University Press.2003, pp. 7-8, 25, 35, 41, 43-44, 51-56.

5. Kumar V, Abbas AK, Fausto N, editors. Robbins and Cotran: Pathologic Basis of Disease. 2005 (7th edition). Philadelphia, PA, Pennsylvania: Elsevier, Saunders.

6. Akbik D, Ghadiri M, Chrzanowski W, Rohanizadeh R. Curcumin as a wound healing agent. Life Sci. 2014; 116(1):1-7.

7. Lim YS, Kwon SK, Park JH, Cho CG, Park SW, Kim WK. Enhanced mucosal healing with curcumin in animal oral ulcer model. Laryngoscope. 2016;126(2):E68-73.

8. Hashemipour MA, Ghasemi AR, Dogaheh MA, Torabi M. Effects of Locally and Systemically Applied n-3 Fatty Acid on Oral Ulcer Recovery Process in Rats. Wounds. 2012; 24(9):258-66.

9. Kaur M , Sable DM , Chowdhery A, Chavan M. A review of omega 3 and it's role in oral diseases. International Journal of Advanced Research. 2016; 4(9): 921-925.

10. Black HS, Rhodes LE. Potential Benefits of Omega-3 Fatty Acids in Non-Melanoma Skin Cancer. Brown L, Rauch B, Poudyal H, eds. Journal of Clinical Medicine. 2016;5(2):23

11. Bhattacharya A, Ghosal S, Bhattacharya SK. Effect of fish oil on offensive and defensive factors in gastric ulceration in rats. Prostaglandins Leukot Essent Fatty Acids. 2006;74(2):109-116.

12. Grimble RF, Howell WM, O'Reilly G, Turner SJ, Markovic O, Hirrell S, East JM, Calder PC. The ability of fish oil to suppress tumor necrosis factor alpha production by peripheral blood mononuclear cells in healthy men is associated with polymorphisms in genes that influence tumor necrosis factor alpha production. The American Journal for Clinical Nutrition. 2002; 76(2):454-459..

13. Gupta P, Ashok L, Naik SR. Assessment of serum interleukin-8 as a sensitive serological marker in monitoring the therapeutic effect of levamisole in recurrent aphthous ulcers: a randomized control study. Indian Journal of Dental Reserch. 2014; 25 (3): 284-289.
14. Alexander JW, Supp DM. Role of Arginine and Omega-3 Fatty Acids in Wound Healing and Infection. Advances in Wound Care. 2014;3(11):682-690.

15. Carbajal Quintana D, Molina Cuevas V, Ravelo Calzado Y, Mas Ferreiro R. Comparison of the protective effects of Omega 3 fatty acids and D- 002 (beeswax alcohols) on the ethanol-induced gastric ulcer in the rat. REDVET. Revista Electrónica de Veterinaria [Internet]. 2013;14(2):1-8.

16. De Castilho TJC, Campos ACL, Mello EV de SL. Effect Of Omega-3 Fatty Acid In The Healing Process Of Colonic Anastomosis In Rats. Arquivos Brasileiros de Cirurgia Digestiva: $\mathrm{ABCD}=$ Brazilian Archives of Digestive Surgery. 2015; 28(4):258-261.

17. Chen X,Thibeault SL.Role of tumor necrosis factor alphain wound repair in human vocal fold fibroblasts, Laryngoscope. 2010;120(9):1819-25.

18. Browning JL, Ngam-ek A, Lawton P, DeMarinis J, Tizard R, Chow EP, Hession C, O'Brine-Greco B, Foley SF, Ware CF. Lymphotoxin beta, a novel member of the TNF family that forms a heteromeric complex with lymphotoxin on the cell surface. Cell. 1993; 72(6): 847-56..

19. Ashcroft GS, Jeong M-J, Ashworth JJ, et al. TNF $\alpha$ is a therapeutic target for impaired cutaneous wound healing. Wound Repair and Regeneration. 2012;20(1):38-49.

20. Tayyebi-Khosroshahi H, Houshyar J, Dehgan-Hesari R, Alikhah H, Vatankhah AM, Safaeian AR, Zonouz NR. Effect of treatment with Omega-3 fatty acids on C-reactive protein and tumor necrosis factor-alfa in hemodialysis patients. Saudi Journal of Kidney Diseases and Transplantation. 2012; 23(3): 500-506.

21. Qazi BS, Tang K, Qazi A. Recent Advances in Underlying Pathologies Provide Insight into Interleukin-8 ExpressionMediated Inflammation and Angiogenesis. International Journal of Inflammation. 2011; 2011: 908468.

22. Matsuyama W, Mitsuyama H, Watanabe M, Oonakahara K, Higashimoto I, Osame M, Arimura K. Effects of omega-3 polyunsaturated fatty acids on inflammatory markers in COPD. Chest Journal. 2005;128(6): 3817-3827.

23. Bradberry JC, Hilleman DE. Overview of Omega-3 Fatty Acid Therapies. Pharmacy and Therapeutics. 2013; 38(11):681-691.

24. Thies F, Nebe-von-Caron G, Powell JR, Yaqoob P, Newsholme EA, Calder PC. Dietary supplementation with eicosapentaenoic acid, but not with other long-chain n-3 
or n-6 polyunsaturated fatty acids, decreases natural killer cell activity in healthy subjects aged $>55 \mathrm{y}$. The American Journal of Clinical Nutrition. 2001; 73(3): 539-548.

25. Kang JX, Weylandt KH. Modulation of inflammatory cytokines by omega-3 fatty acids. Subcellular Biochemistry 2008;49:133-143.

26. Hankenson KD, Watkins BA, Schoenlein IA, Allen KG, Turek JJ. Omega-3 fatty acids enhance ligament fibroblast collagen formation in association with changes in interleukin-6 production. Proceedings of the Society for Experimental Biology and Medicine. 2000; 223(1): 88-95.

27. Gercek A, Yildirim O, Konya D, Bozkurt S, Ozgen S, Kilic T, Sav A, Pamir N. Effects of parenteral fish-oil emulsion
(Omegaven) on cutaneous wound healing in rats treated with dexamethasone. Journal of Parenteral and Enteral Nutrition. 2007; 31 (3):161-166.

28. Cardoso CR, Souza MA, Ferro EA, Favoreto S Jr, Pena JD.I nfluence of topical administration of n-3 and n-6 essential and $\mathrm{n}-9$ nonessential fatty acids on the healing of cutaneous wounds, Wound Repair Regen.2004;12(2):35-243.

29. Terkelsen LH, Eskild-Jensen A, Kjeldsen H, et al: Topical application of cod liver oil ointment accelerates wound healing: an experimental study in wounds in the ears of hairless mice. Scandinavian Journal of Plastic and Reconstructive Surgery and Hand Surgery. 2000; 34(1):15-20. 